\title{
Compensating of added mass terms in dynamically positioned surface vehicles: A continuous robust control approach
}

\author{
Baris Bidiklia,b $^{\mathrm{a},}$ Enver Tatlicioglu ${ }^{\mathrm{a}, *}$, Erkan Zergeroglu $^{\mathrm{c}}$

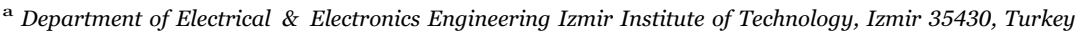 \\ b Department of Mechatronics Engineering, Faculty of Engineering and Architecture, Izmir Katip Celebi University, Izmir 35620, Turkey \\ ${ }^{\mathrm{c}}$ Department of Computer Engineering, Gebze Technical University, 41400 Gebze, Kocaeli, Turkey
}

\section{A R T I C L E I N F O}

\section{Keywords:}

Surface vessel

Positioning control

Added mass

Robust control

\begin{abstract}
A B S T R A C T
In this work, we provide a tracking controller formulation for dynamically positioned surface vessels with an asymmetric added mass terms that affects the overall system dynamics at the acceleration level. Specifically a novel continuous robust controller is proposed for surface vessels that in addition to unstructured uncertainties in its dynamics, contains added mass effects in its inertia matrix. The proposed controller compensates the overall system uncertainties while ensuring asymptotic tracking by utilizing the knowledge of the leading principal minors of the input gain matrix. Stability of the closed-loop system and asymptotic convergence are proven via Lyapunov based approaches. Simulation studies are also presented to illustrate the viability of the proposed method.
\end{abstract}

\section{Introduction and system model}

The mathematical model for a dynamically positioned fully actuated 3 degree of freedom (dof) surface vessel is commonly represented by Fossen (2011,1994,2002), Skjetne et al. (2004), Ihle et al. (2006).

$M_{s} \dot{v}+C_{s} v+D_{s} v=\tau$

$\dot{x}=R v$

where $x(t) \triangleq\left[x_{p}, y_{p}, \psi\right]^{T} \in \mathbb{R}^{3}$ is the position vector that contains translational positions $x_{p}(t), y_{p}(t) \in \mathbb{R}$ in $\mathrm{X}-$ and $\mathrm{Y}-$ directions, respectively, and the yaw angle of the ship $\psi(t) \in \mathbb{R}$, $v(t)=[u, v, \dot{\psi}]^{T} \in \mathbb{R}^{3}$ is the body-fixed linear and angular velocity vector. Also in (1), $M_{s}(\psi), C_{s}\left(v, v_{r}\right), D_{s}\left(v, v_{r}\right) \in \mathbb{R}^{3 \times 3}$ represent inertia matrix, centripetal and Coriolis forces, hydrodynamic damping terms, respectively, $v_{r}(t) \in \mathbb{R}^{3}$ is the relative velocity between the fluids and the vessel, and the control input vector is represented by $\tau(t) \in \mathbb{R}^{3}$. In (2), $R(\psi) \in S O$ (3) is the rotation matrix that has the form

$R(\psi)=\left[\begin{array}{lll}\cos (\psi) & -\sin (\psi) & 0 \\ \sin (\psi) & \cos (\psi) & 0 \\ 0 & 0 & 1\end{array}\right]$.

While the mathematical model in (1) and (2) are utilized in almost all past work, as detailed in Fossen (1994,2002), Skjetne et al. (2004), and Fossen and Strand (1999), during the cruise, the motion of the surface vessel effects all the flow, resulting in vibrations with different amplitudes to occur on various parts of the flow. It is important to note that motion in one direction cause forces not only in the same direction but also in other directions (Newman, 1977; Lewis, 1989). This situation results as pressure effects and moments acting on different parts of the surface vessels and submarines which causes additional force and thus has an influence on the acceleration of surface vessels and submarines. For precise control design, this effect, referred as the added mass, is required to be represented in the dynamic model. There are different conventions (Skjetne et al., 2004; Fossen and Strand, 1999; Kim et al., 2007) on how to represent the added mass effects in the dynamic model. To name a few: In Fossen and Strand (1999), after using inertial velocity as the velocity state, the added mass effects are represented inside the system's inertia matrix. Following the convention given in Fossen and Strand (1999), in this work, the added mass terms are considered to be affecting the system dynamics at the acceleration level (i.e., inertial velocity is chosen as the velocity state). As a result, the inertia matrix of the surface vessel in (1) is obtained as (Fossen, 1994)

$M_{s}=M_{R B}+M_{A}$

where $M_{R B}(\psi) \in \mathbb{R}^{3 \times 3}$ represents the positive definite, symmetric rigid body inertia matrix and $M_{A}(\psi) \in \mathbb{R}^{3 \times 3}$ represents the added mass inertia matrix. The entries of added mass inertia matrix represented by $M_{A_{i j}}$ denote the mass associated with a force on the body in the $i$ th direction due to a unit acceleration in the $j$ th direction (Techet, 2015).

\footnotetext{
* Corresponding author.

E-mail address: enver@iyte.edu.tr (E. Tatlicioglu).
} 
For a detailed representation of the mathematical model reader can refer to Lee et al. (2008).

As noted in Fossen (1994) the inertia matrix due to added mass is not necessarily symmetric. After summed with the symmetric $M_{R B}(\psi)$, the overall inertia matrix $M_{s}(\psi)$ of the system loses its symmetry. The asymmetric terms in the inertia matrix, when not appropriately dealt with, may result in degradation of the controller performance, and even instability (Lee et al., 2008, 2008). Therefore the main challenge of added mass effects are due to its asymmetric nature. From a control design perspective, the symmetric nature of the inertia matrix is extremely useful especially when constructing quadratic terms in the Lyapunov candidate function.

To our best knowledge, there are only a few control design works that considered asymmetric added mass in the inertia matrix. In Do and Pan (2005), Do and Pan considered the case where the symmetry of the inertia matrix was removed for underactuated surface vehicles. Robust and adaptive type controllers were proposed for the fully actuated surface vehicles in Lee et al. (2008) and Lee et al. (2008), respectively. The aforementioned controllers were designed based on Lyapunov-type analysis methods, and were able to achieve only the ultimate boundedness of the tracking error signals.

In this work, a surface vessel having asymmetric inertia matrix is considered and output tracking control is aimed. The asymmetry of the inertia matrix is to be dealt with a matrix decomposition which while proposing a solution to the asymmetric inertia matrix by introducing a symmetric inertia-like matrix causes the control input to be premultiplied first with an uncertain unity upper triangular matrix and then with a known diagonal matrix. This is then addressed via the control design and accompanying stability analysis where first boundedness of all the closed-loop signals are demonstrated. Then via the use of two lemmas semi-global asymptotic stability of the tracking error is proven. Numerical simulations are then performed to demonstrate the viability of the proposed robust control strategy.

\section{Open-loop error system development}

In an attempt to obtain a compact representation of the mathematical model of the surface vessel in (1) and (2), the time derivative of (2) is taken

$\ddot{x}=\dot{R} v+R \dot{v}$

which includes the time derivative of the rotation matrix that can be obtained as

$\dot{R}=R S_{3}$

with $S_{3}(\dot{\psi}) \in \mathbb{R}^{3 \times 3}$ being a skew-symmetric matrix defined as

$S_{3} \triangleq \dot{\psi}\left[\begin{array}{lll}0 & -1 & 0 \\ 1 & 0 & 0 \\ 0 & 0 & 0\end{array}\right]$.

After substituting (1) and (6) into (5), it is easy to obtain

$\ddot{x}=R M_{s}^{-1} \tau-R\left[M_{s}^{-1}\left(C_{s}+D_{s}\right)-S_{3}\right] v$.

In order to ease the presentation of the subsequent development, the right-hand side of (8) can be rewritten as

$\ddot{x}=h+G \tau$

where $h(x, \dot{x}) \in \mathbb{R}^{3}$ and $G(x, \dot{x}) \in \mathbb{R}^{3 \times 3}$ are defined as

$h \triangleq-R\left[M_{s}^{-1}\left(C_{s}+D_{s}\right)-S_{3}\right] v$

$G \triangleq R M_{s}^{-1}$.

It is assumed that $G(x, \dot{x})$ being a real matrix with non-zero leading principle minors by utilizing the assumption that $M_{s}(\psi)$ has full rank. This allows the utilization of the matrix decomposition in Costa et al. (2003), Morse (1993) to yield
$G=S D U$

where $S(x, \dot{x}), D$ and $U(x, \dot{x}) \in \mathbb{R}^{3 \times 3}$ denote a symmetric positive definite matrix, a diagonal matrix with entries being \pm 1 , and a unity upper triangular matrix, respectively. When the above matrix decomposition is applied to the simulation model taken from Skjetne et al. (2004), $D$ came out to be an identity matrix. Despite this, the derivations will be made for the general case where $D$ is assumed to be available for the control design (see Chen et al., 2008 for a similar type of assumption).

From (9), via utilizing the assumption that the leading principal minors of $G$ are non-zero, it is easy to obtain

$\tau=G^{-1}(\ddot{x}-h)$.

After taking the time derivative of (9), substituting (12) and (13), and then performing straightforward mathematical manipulations yield

$\dddot{x}=\varphi+S D U \dot{\tau}$

where $\varphi(x, \dot{x}, \ddot{x}) \in \mathbb{R}^{3}$ is an auxiliary vector that is defined as

$\varphi \triangleq \dot{h}+\dot{G} G^{-1}(\ddot{x}-h)$.

At this point, $M(x, \dot{x}) \in \mathbb{R}^{3 \times 3}$ is defined as the inverse of $S(x, \dot{x})$. Since $S(x, \dot{x})$ obtained from the matrix decomposition is symmetric and positive definite, so is $M(x, \dot{x})$. Furthermore, $M(x, \dot{x})$ satisfies the following inequalities

$\underline{m}\|\chi\|^{2} \leq \chi^{T} M(x, \dot{x}) \chi \leq \bar{m}(x, \dot{x})\|\chi\|^{2} \forall \chi \in \mathbb{R}^{3 \times 1}$

where $\underline{m} \in \mathbb{R}$ and $\bar{m}(x, \dot{x}) \in \mathbb{R}$ represent a positive bounding constant and a positive non-decreasing function, respectively.

Pre-multiplying both sides of (14) with $M(x, \dot{x})$ yields

$M \dddot{x}=f+D U i$

where $f(x, \dot{x}, \ddot{x}) \triangleq M \varphi \in \mathbb{R}^{3}$.

Ensuring that the translational positions and the yaw angle would track a given reference trajectory while, at the same time, ensuring the boundedness of all the signals under the closed-loop operation consists of our main control objectives. The control design is based on availability of $x(t)$ and $\dot{x}(t)$ (i.e., full-state feedback).

In order to quantify the tracking control objective, the output tracking error, $e_{1}(t) \in \mathbb{R}^{3}$, is defined as the difference between the reference trajectory and the position of the surface vessel as

$e_{1} \triangleq x_{d}-x$

where $x_{d}(t) \in \mathbb{R}^{3}$ is the reference trajectory chosen smooth enough in the sense that

$x_{d}(t) \in C^{3} \quad$ and $\quad x_{d}^{(i)}(t) \in \mathcal{L}_{\infty}, \quad i=0,1,2,3$.

In order to eliminate the higher order terms from our stability analysis (i.e., only first order time derivatives to appear in the time derivative of the Lyapunov function), an auxiliary error signal, denoted by $e_{2}(t) \in \mathbb{R}^{3}$, and a filtered error term, denoted by $r(t) \in \mathbb{R}^{3}$, are defined as follows

$e_{2} \triangleq \dot{e}_{1}+e_{1}$

$r \triangleq \dot{e}_{2}+\alpha e_{2}$

where $\alpha \in \mathbb{R}^{3 \times 3}$ is a positive definite, diagonal, constant gain matrix. After premultiplying the time derivative of (21) with $M$, the following expression can be obtained

$M \dot{r}=M\left(\dddot{x}_{d}+\ddot{e}_{1}+\alpha \dot{e}_{2}\right)-f-D U \dot{\tau}$

where (17), (18) and (20) were utilized. To obtain a compact form for the right-hand side of (22), we define an auxiliary function $N\left(x, \dot{x}, \ddot{x}, x_{d}, \dot{x}_{d}, \ddot{x}_{d}, \dddot{x}_{d}, t\right) \in \mathbb{R}^{3}$ as follows

$N \triangleq M\left(\dddot{x}_{d}+\ddot{e}_{1}+\alpha \dot{e}_{2}\right)-f+e_{2}+\frac{1}{2} \dot{M} r$. 
In view of (23), the expression in (22) can now be reformulated as

$M \dot{r}=-\frac{1}{2} \dot{M} r-e_{2}-D U \dot{\tau}+N$.

In the right-hand side of (24), [via the definition of $N$ in (23)] the terms $-\frac{1}{2} \dot{M} r$ and $-e_{2}$ are introduced to cancel some terms that will appear in the time derivative of the subsequently designed Lyapunov function.

The auxiliary function $N$ is now segregated into two auxiliary functions in a way by grouping the terms that can be bounded by known constants, and the terms that can be bounded by error signals. Mathematically, $\bar{N}(t), \widetilde{N}(t) \in \mathbb{R}^{3}$ are defined as

$\left.\bar{N} \triangleq N\right|_{x=x_{d}, \dot{x}=\dot{x}_{d}, \ddot{x}=\ddot{x}_{d}}$

$\widetilde{N} \triangleq N-\bar{N}$.

Finally, after substituting the above definitions, the error dynamics in (24) can be rearranged as

$M \dot{r}=-\frac{1}{2} \dot{M} r-e_{2}-D U \dot{\tau}+\widetilde{N}+\bar{N}$.

\section{Controller formulation}

Based on the open-loop error system in (27) and the subsequent stability analysis, the control input $\tau(t)$ is designed to have the following form

$\tau=D K\left[e_{2}(t)-e_{2}\left(t_{0}\right)+\alpha \int_{t_{0}}^{t} e_{2}(\sigma) d \sigma\right]+D \Pi$

where the auxiliary signal $\Pi(t) \in \mathbb{R}^{3}$ is generated according to the update law

$\dot{\Pi}(t)=C \operatorname{Sgn}\left(e_{2}(t)\right) \quad$ with $\Pi\left(t_{0}\right)=0_{3}$.

In (28) and (29), $K, C \in \mathbb{R}^{3 \times 3}$ are positive definite, diagonal, constant gain matrices, $0_{3} \in \mathbb{R}^{3}$ is a vector of zeros and $\operatorname{Sgn}(\cdot) \in \mathbb{R}^{3}$ is the vector signum function. The control gain is chosen as $K=I_{3}+k_{p} I_{3}+\operatorname{diag}\left\{k_{d, 1}, k_{d, 2}, 0\right\}$ where $k_{p}, k_{d, 1}, k_{d, 2} \in \mathbb{R}$ are positive, constant controller gains, the notation $\operatorname{diag}\{\cdot\}$ represents a diagonal matrix, and $I_{3} \in \mathbb{R}^{3 \times 3}$ is the standard identity matrix. Finally, after substituting the time derivative of the control input in (28) into (27) and then adding and subtracting $D K r(t)$, following closed-loop error system for $r(t)$ is obtained

$M \dot{r}=-\frac{1}{2} \dot{M} r-e_{2}-K r+\widetilde{N}+\bar{N}-D\left(U-I_{3}\right) D K r-\operatorname{DUDCSgn}\left(e_{2}\right)$

where the fact that $D D=I_{3}$ was also utilized.

We would like to draw attention to the last two terms of (30) by investigating them separately before proceeding with the stability analysis. It is highlighted that, our investigation on these two terms rely on the fact that $U$ is a unity upper triangular matrix and thus $\left(U-I_{3}\right)$ is a strictly upper triangular matrix.

Notice that, we can rewrite $D\left(U-I_{3}\right) D K r$ as follows

$D\left(U-I_{3}\right) D K r=\left[\begin{array}{c}\Lambda_{1} \\ \Lambda_{2} \\ 0\end{array}\right]+\left[\begin{array}{c}\Phi_{1} \\ \Phi_{2} \\ 0\end{array}\right]$

where $\Lambda_{1}(t), \Lambda_{2}(t), \Phi_{1}(t), \Phi_{2}(t) \in \mathbb{R}$ are auxiliary signals being defined as

$\Lambda_{1} \triangleq d_{1} d_{2} k_{2} \widetilde{U}_{1,2} r_{2}+d_{1} d_{3} k_{3} \widetilde{U}_{1,3} r_{3}$

$\Lambda_{2} \triangleq d_{2} d_{3} k_{3} \widetilde{U}_{2,3} r_{3}$

$\Phi_{1} \triangleq d_{1} d_{2} k_{2} \bar{U}_{1,2} r_{2}+d_{1} d_{3} k_{3} \bar{U}_{1,3} r_{3}$

$\Phi_{2} \triangleq d_{2} d_{3} k_{3} \bar{U}_{2,3} r_{3}$ with $\bar{U}_{1,2}(t), \bar{U}_{1,3}(t), \bar{U}_{2,3}(t), \widetilde{U}_{1,2}(t), \widetilde{U}_{1,3}(t), \widetilde{U}_{2,3}(t) \in \mathbb{R}$ are defined as

$\left.\bar{U}_{i, j} \triangleq U_{i, j}\right|_{x=x_{d}, \dot{x}=\dot{x}_{d}}$

$\widetilde{U}_{i, j} \triangleq U_{i, j}-\bar{U}_{i, j}$

where $U_{i, j}(x, \dot{x}) \in \mathbb{R}$ are the entries of $U(x, \dot{x})$. Notice from (33) that $\Lambda_{2}(t)$ depends on $k_{3}$, and from (32), $\Lambda_{1}(t)$ depends on $k_{3}$ and $k_{2}$. Similarly, from (35), $\Phi_{2}(t)$ depends on $k_{3}$, and from (34), $\Phi_{1}(t)$ depends on $k_{3}$ and $k_{2}$.

On the other hand, the term $\operatorname{DUDCSgn}\left(e_{2}\right)$ can be decomposed as

$\operatorname{DUDCSgn}\left(e_{2}\right)=\left[\begin{array}{l}\Psi \\ 0\end{array}\right]+\Theta$

with two auxiliary signals $\Psi(t) \in \mathbb{R}^{2}$ and $\Theta(t) \in \mathbb{R}^{3}$ are defined as

$\left[\begin{array}{l}\Psi \\ 0\end{array}\right]=D(U-\bar{U}) D C \operatorname{Sgn}\left(e_{2}\right)$

\section{$\Theta \triangleq D \bar{U} D C \operatorname{Sgn}\left(e_{2}\right)$}

where $\bar{U}\left(x_{d}, \dot{x}_{d}\right) \triangleq U \mathrm{I}_{x=x_{d}, \dot{x}=\dot{x}_{d}} \in \mathbb{R}^{3 \times 3}$ is a function of reference trajectory and its time derivative, and $\Psi_{i}(t) \in \mathbb{R}, i=1,2$ and $\Theta_{i}(t) \in \mathbb{R}, i=1,2,3$, are defined as

$\Psi_{i}=d_{i} \sum_{j=i+1}^{3} d_{j} C_{j} \widetilde{U}_{i, j} \operatorname{sgn}\left(e_{2, j}\right)$

$\Theta_{i}=d_{i} \sum_{j=i}^{3} d_{j} C_{j} \bar{U}_{i, j} \operatorname{sgn}\left(e_{2, j}\right)$.

Remark 1. The Mean Value Theorem (Khalil and Systems, 2002) can be utilized to develop the following upper bounds

$\|\widetilde{N}\| \leq \rho_{\widetilde{N}}(\|z\|)\|z\|$

$\left|\widetilde{U}_{i, j}\right| \leq \rho_{i, j}(\|z\|)\|z\|$

where $\rho_{\bar{N}}, \rho_{i, j} \in \mathbb{R}$ are non-negative, globally invertible, nondecreasing functions of their arguments, and $z(t) \in \mathbb{R}^{9}$ is defined by

$z \triangleq\left[\begin{array}{lll}e_{1}^{T} & e_{2}^{T} & r^{T}\end{array}\right]^{T}$.

In view of (19) (i.e., the smoothness of the reference trajectory), it can be seen from (25) and (36) that $\bar{N}(t)$ and $\bar{U}_{i, j}(t)$ can be bounded by known constants in the sense that

$\left|\bar{N}_{i}(t)\right| \leq \zeta_{\bar{N}_{i}}$

$\left|\bar{U}_{i, j}(t)\right| \leq \zeta_{D_{i, j}}$

where $\zeta_{\bar{N}_{i}}, \zeta_{\bar{U}_{i, j}} \in \mathbb{R}$ are positive bounding constants. Based on (32)(35), following upper bounds can be obtained for $i=1,2$

$\left|\Lambda_{i}\right| \leq \rho_{\Lambda_{i}}(\|z\|)\|z\|$

$\left|\Phi_{i}\right| \leq \zeta_{\Phi_{i}}\|z\|$

$\left|\Psi_{i}\right| \leq \leq \rho_{\Psi_{i}}(\|z\|)\|z\|$

and for $i=1,2,3$

$\left|\Theta_{i}\right| \leq \zeta_{\Theta_{i}}$

where (43)-(47) were utilized. From (51), it is easy to see that $\|\Theta\| \leq \zeta_{\Theta}$ is satisfied for some positive bounding constant $\zeta_{\Theta} \in \mathbb{R}$, and from (48)(50), we have

$\left|\Lambda_{i}\right|+\left|\Phi_{i}\right|+\left|\Psi_{i}\right| \leq \rho_{i}(\|z\|)\|z\|$

where $\rho_{i}(\|z\|) \in \mathbb{R} i=1,2$, are non-negative, globally invertible, nondecreasing functions satisfying

$\rho_{\Lambda_{i}}+\rho_{\Psi_{i}}+\zeta_{\Phi_{i}} \leq \rho_{i}$ 


\section{Stability analysis}

In this section, initially, the boundedness of the error signals under the closed-loop operation will be proven by using a Lyapunov type analysis. Then, a lemma will be presented and an upper bound for the integral of the absolute values of the entries of the time derivative of $e_{2}(t)$ will be obtained by using the boundedness result. In order to prove the non-negativity of a Lyapunov-like function, this upper bound will be utilized in another lemma that will be used in our final analysis which proves asymptotic stability of the overall closed-loop system.

Theorem 1. For the mathematical model of the surface vessel in (1) and (2), the controller in (28) and (29) guarantee the boundedness of the error signals in (18), (20) and (21) provided that the controller gains $k_{d, 1}, k_{d, 2}$ and $k_{p}$ are chosen large enough compared to the initial conditions of the system and the following condition is satisfied

$\lambda_{\min }(\alpha) \geq \frac{1}{2}$

where the notation $\lambda_{\min }(\alpha)$ denotes the minimum eigenvalue of the gain matrix $\alpha$.

Proof. The non-negative function $V_{b}(z) \in \mathbb{R}$ is defined as

$V_{b} \triangleq \frac{1}{2} e_{1}^{T} e_{1}+\frac{1}{2} e_{2}^{T} e_{2}+\frac{1}{2} r^{T} M r$.

In view of (16), the Lyapunov function in (55) can be lower and upper bounded in the following manner

$\frac{1}{2} \min \{1, \underline{m}\}\|z\|^{2} \leq V_{b}(z) \leq \frac{1}{2} \max \{1, \bar{m}(\|z\|)\}\|z\|^{2}$

where $z(t)$ was defined in (45), and the terms $\underline{m}, \bar{m}(\|z\|)$ were introduced in (16). The time derivative of (55) can be upper bounded as

$\dot{V}_{b} \leq-\beta_{1} V_{b}+\beta_{2}$

where $\beta_{1}, \beta_{2} \in \mathbb{R}$ are positive constants. From (55) and (57), we can conclude that $V_{b}(t) \in \mathcal{L}_{\infty}$, therefore $e_{1}(t), e_{2}(t)$ and $r(t)$ are uniformly ultimately bounded. Standard signal chasing arguments can then be utilized to prove that all the signals remain bounded under the closedloop operation. $\square$

Lemma 1. Provided that $e_{2}(t)$ and $\dot{e}_{2}(t)$ are bounded, the following expression for the upper bound of the integral of the absolute value of the $i$ - - th entry of $\dot{e}_{2}(t) i=1,2,3$ can be obtained

$\int_{t_{0}}^{t}\left|\dot{e}_{2, i}(\sigma)\right| d \sigma \leq \gamma_{1}+\gamma_{2} \int_{t_{0}}^{t}\left|e_{2, i}(\sigma)\right| d \sigma+\left|e_{2, i}\right|$

where $\gamma_{1}, \gamma_{2} \in \mathbb{R}$ are some positive bounding constants.

Proof. The proof is similar to that of the one given in Stepanyan and Kurdila (2009), its most general form can also be found in Appendix B of Bidikli et al. (2013). It should be noted that proof in Bidikli et al. (2013) can be used precisely for the above mentioned lemma by using 2 instead of $n$ given in the mentioned study. $\square$

Following decomposition is essential for the proof of Lemma 2.

Remark 2. Notice that, as a result of the fact that $\bar{U}(t)$ being unity upper triangular, $\Theta(t)$ in (40) can be rewritten as

$\Theta=\left(I_{3}+\Omega\right) C \operatorname{Sgn}\left(e_{2}\right)$

where $\Omega(t) \triangleq D\left(\bar{U}-I_{3}\right) D \in \mathbb{R}^{3 \times 3}$ is a strictly upper triangular matrix. Since it is a function of the reference trajectory and its time derivatives, its entries, denoted by $\Omega_{i, j}(t) \in \mathbb{R}$, are bounded in the sense that

$\left|\Omega_{i, j}\right| \leq \zeta_{\Omega_{i, j}}$

where $\zeta_{\Omega_{i, j}} \in \mathbb{R}$ are positive bounding constants.

Lemma 2. Consider the term
$L \triangleq r^{T}\left(\bar{N}-\left(I_{3}+\Omega\right) C \operatorname{Sgn}\left(e_{2}\right)\right)$.

Provided that the entries of the control gain matrix $C$ are chosen to satisfy

$C_{3} \geq \zeta_{N_{3}}\left(1+\frac{\gamma_{2}}{\alpha_{3}}\right)$

$C_{2} \geq\left(\zeta_{\bar{N}_{2}}+\zeta_{\Omega_{2,3}} C_{3}\right)\left(1+\frac{\gamma_{2}}{\alpha_{2}}\right)$

$C_{1} \geq\left(\zeta_{N_{1}}+\zeta_{\Omega_{1,2}} C_{2}+\zeta_{\Omega_{1,3}} C_{3}\right)\left(1+\frac{\gamma_{2}}{\alpha_{1}}\right)$

in order, then it can be concluded that

$\int_{t_{0}}^{t} L(\sigma) d \sigma \leq \zeta_{L}$

where $\zeta_{L} \in \mathbb{R}$ is a positive bounding constant defined as

$\zeta_{L} \triangleq \gamma_{1} \sum_{i=1}^{2} \sum_{j=i+1}^{3} \zeta_{\Omega_{i, j}} C_{j}+\gamma_{1} \sum_{i=1}^{3} \zeta_{\overline{N_{i}}}+\sum_{i=1}^{3} C_{i}\left|e_{2, i}\left(t_{0}\right)\right|$.

Proof. Its most general form can be found in Appendix C of Bidikli et al. (2013). It should be noted that proof in Bidikli et al. (2013) can be used precisely for the above mentioned lemma by using 3 instead of $m$ and 1, 2 instead of $i$ given in the mentioned study. $\square$

Theorem 2. Given the dynamic model of the surface vessel in (1) and (2), the continuous robust controller of (28) and (29) ensures the tracking error signal $e_{1}(t)$ and the auxiliary error signals $e_{2}(t)$ and $r(t)$ converge to zero asymptotically in the sense that

$\left\|e_{1}(t)\right\|,\left\|e_{2}(t)\right\|,\|r(t)\| \rightarrow 0$ ast $\rightarrow+\infty$

provided that $\alpha$ is chosen to satisfy (54), the entries of $C$ are chosen to satisfy (62)-(64), and $k_{p}, k_{d, 1}, k_{d, 2}$ are chosen large enough.

Proof. Let the auxiliary function $P(t) \in \mathbb{R}$ be defined as follows

$P \triangleq \zeta_{L}-\int_{t_{0}}^{t} L(\sigma) d \sigma$

where the terms $\zeta_{L}$ and $L(t)$ were defined in (66) and (61), respectively. When the entries of the control gain matrix $C$ are chosen to satisfy (62)-(64), from the proof of Lemma 2 given in Appendix C of Bidikli et al. (2013), we can conclude that $P(t)$ is non-negative.

At this stage, consider the Lyapunov function $V(s, t) \in \mathbb{R}$ defined as $V \triangleq V_{b}+P$

where $s(t) \in \mathbb{R}^{10}$ is defined as

$s \triangleq\left[z^{T} \sqrt{P}\right]^{T}$

and $V_{b}(t) \in \mathbb{R}$ was defined in (55). By utilizing (16), the Lyapunov function in (69) can be lower and upper bounded in the following form $W_{1}(s) \leq V(s, t) \leq W_{2}(s)$

where $W_{1}(s), W_{2}(s) \in \mathbb{R}$ are defined as

$W_{1} \triangleq \lambda_{2}\|s\|^{2}, \quad W_{2} \triangleq \lambda_{3}(\|s\|)\|s\|^{2}$

with $\lambda_{2} \triangleq \frac{1}{2} \min \{1, \underline{m}\}$ and $\lambda_{3} \triangleq \max \left\{1, \frac{1}{2} \bar{m}(\|z\|)\right\}$.

Taking the time derivative of $V(t)$, utilizing the time derivative of (65), canceling common terms and following similar steps to that of proof of Theorem 1 yields

$$
\begin{aligned}
\dot{V}= & -e_{1}^{T} e_{1}+e_{1}^{T} e_{2}-e_{2}^{T} \alpha e_{2}-r^{T} r \\
& +\left[r^{T} \widetilde{N}-k_{p} r^{T} r\right] \\
& +\left[-\sum_{i=1}^{m-1} r_{i}\left(\Lambda_{i}+\Psi_{i}+\Phi_{i}\right)-\sum_{i=1}^{2} k_{d, i} r_{i}^{2}\right]
\end{aligned}
$$

which can be rearranged to have the following form 


$$
\begin{gathered}
\dot{V} \leq \quad-\frac{1}{2}\left\|e_{1}\right\|^{2}-\left(\lambda_{\min }(\alpha)-\frac{1}{2}\right)\left\|e_{2}\right\|^{2}-r^{T} r \\
+\frac{\rho_{N}^{2}(\|z\|)}{4 k_{p}}\|z\|^{2}+\sum_{i=1}^{2} \frac{\rho_{i}^{2}(\|z\|)}{4 k_{d, i}}\|z\|^{2} \\
\leq-\left(\lambda_{4}-\frac{\rho_{N}^{2}(\|z\|)}{4 k_{p}}-\sum_{i=1}^{2} \frac{\rho_{i}^{2}(\|z\|)}{4 k_{d, i}}\right)\|z\|^{2}
\end{gathered}
$$

where $\lambda_{4} \triangleq \min \left\{\frac{1}{2}, \lambda_{\min }(\alpha)-\frac{1}{2}\right\}$. When the controller gains $k_{p}, k_{d, 1}, k_{d, 2}$ are selected large enough such that the regions defined by $\mathcal{D}_{z} \triangleq\{z:\|z\| \leq \mathcal{R}\}$ and $\mathcal{D}_{s} \triangleq\{s:\|s\| \leq \mathcal{R}\}$ with $\mathcal{R}$ being defined as

$\mathcal{R}=\min \left\{\rho_{\bar{N}}^{-1}\left(2 \sqrt{k_{p} \frac{1-\beta}{3}}\right), \rho_{1}^{-1}\left(2 \sqrt{k_{d, i} \frac{1-\beta}{3}}\right), \rho_{2}^{-1}\left(2 \sqrt{k_{d, i} \frac{1-\beta}{3}}\right)\right\}$

are non-empty, from (75) and the definition of $s$, one can restate

$\dot{V} \leq-\beta\|z\|^{2}=-W(s) \forall s \in \mathcal{D}_{s}$

where $\beta \in \mathbb{R}$ is a positive constant that satisfies $0 \leq \beta<1$. From the definition of (69) and (77), it is obvious that $V(t) \in \mathcal{L}_{\infty}$, also from the proof of Theorem 1 and outcome of standard linear analysis methods, we can conclude that all the signals in the closed-loop error system are bounded and furthermore, from the boundedness of $\dot{W}(s)$, we can state that $W(s)$ is uniformly continuous.

Based on the definition of $\mathcal{D}_{s}$, another region, $\mathcal{S}$, can be defined in the following form

$$
\begin{aligned}
\mathcal{S} \triangleq & \left\{s \in \mathcal{D}_{s}: W_{2}(s)<\lambda_{3}\left(\rho_{\bar{N}}^{-1}\left(2 \sqrt{k_{p} \frac{1-\beta}{3}}\right)\right)^{2}\right\} \\
& \cap\left\{s \in \mathcal{D}_{s}: W_{2}(s)<\lambda_{3}\left(\rho_{1}^{-1}\left(2 \sqrt{k_{d, 1} \frac{1-\beta}{3}}\right)\right)^{2}\right\} \\
& \cap\left\{s \in \mathcal{D}_{s}: W_{2}(s)<\lambda_{3}\left(\rho_{2}^{-1}\left(2 \sqrt{k_{d, 2} \frac{1-\beta}{3}}\right)\right)^{2}\right\} .
\end{aligned}
$$

A direct application of Theorem 8.4 in Khalil (2002) can be used to prove that $\|z(t)\| \rightarrow 0$ as $t \rightarrow+\infty \forall s\left(t_{0}\right) \in \mathcal{S}$. Based on the definition of $z(t)$, it is easy to show that $\left\|e_{1}(t)\right\|,\left\|e_{2}(t)\right\|,\|r(t)\| \rightarrow 0$ as $t \rightarrow+\infty \forall s\left(t_{0}\right) \in \mathcal{S}$. Note that the region of attraction can be made arbitrarily large to include any initial conditions by choosing the controller gains $k_{p}, k_{d, 1}$ and $k_{d, 2}$. This fact implies that the stability result obtained by proposed method is semi-global. $\square$

\section{Numerical simulation results}

The performance of the proposed robust controller was demonstrated via a numerical simulation. The mathematical model of the surface vessel in (1) was utilized with the inertia matrix that have following rigid body and added mass inertia parts (Skjetne et al., 2004)

$$
\begin{aligned}
M_{R B} & =\left[\begin{array}{lll}
m & 0 & 0 \\
0 & m & m x_{g} \\
0 & m x_{g} & I_{z}
\end{array}\right] \\
M_{A} & =\left[\begin{array}{lll}
X_{\dot{u}} & 0 & 0 \\
0 & -Y_{\dot{v}} & -Y_{\dot{r}} \\
0 & -N_{\dot{v}} & N_{\dot{r}}
\end{array}\right] .
\end{aligned}
$$

In the above inertia matrix, constant terms $Y_{\dot{r}}$ and $N_{\dot{v}}$ are selected as

$Y_{\dot{r}}=0.0, N_{\dot{v}}=-1.0$

that yielded an asymmetric inertia matrix. The Coriolis matrix has the following form
$C_{s}=\left[\begin{array}{ccc}0 & 0 & c_{2} \\ 0 & 0 & -c_{1} \\ -c_{2} & c_{1} & 0\end{array}\right]$

where its entries are given as

$c_{1}=m u+\left(-X_{\dot{u}} u_{r}\right)$

$c_{2}=-m\left(x_{g} \dot{\psi}+v\right)+Y_{\dot{v}} v_{r}+0.5\left(Y_{\dot{r}}+N_{\dot{v}}\right) \dot{\psi}$

where the numerical parameters are given as

$m=23.8, x_{g}=0.046, I_{z}=1.760$

$X_{\dot{u}}=-2.0, Y_{\dot{v}}=-10.0, N_{\dot{r}}=-1.0$.

The damping matrix has the following form

$D_{s}=\left[\begin{array}{ccc}d_{11} & 0 & 0 \\ 0 & d_{22} & d_{23} \\ 0 & d_{32} & d_{33}\end{array}\right]$

with its entries defined as

$d_{11}=-X_{u}-X_{|u| u}\left|u_{r}\right|-X_{\text {иии }} u_{r}^{2}$

$d_{22}=-Y_{v}-Y_{|v| v}\left|u_{r}\right|-Y_{|r| v}|\psi \dot{\psi}|$

$d_{33}=-N_{r}-Y_{|v| v}\left|u_{r}\right|-Y_{|r| v}|\dot{\psi}|$

$d_{23}=-Y_{r}-Y_{|v| r \mid}\left|u_{r}\right|-Y_{|r| v}|\dot{\psi}| d_{32}=-N_{v}-N_{|v| v}\left|v_{r}\right|-N_{|r| v}|\psi \dot{\psi}|$

where $v_{r}=[3,0,0]^{T}$ and the other numerical parameters are given as

$X_{u}=-0.72253, X_{|u| u}=-1.32742, X_{\text {иии }}-5.86643$

$Y_{v}=-0.88965, Y_{|v| v}=-36.47287, Y_{|r| v}=-0.805$

$N_{r}=-1.900, Y_{r}=-7.250, Y_{|v| r}=-0.845$

$N_{v}=0.03130, N_{|v| v}=3.95645, N_{|r| v}=0.130$.

All the other system parameters are obtained from the experimental results at (Skjetne et al., 2004). We would like to highlight that these model parameters were used only for simulation purposes and they were not utilized in the control input. The surface vessel was considered to be initially at rest at $x(0)=\left[0.1,1,-\frac{\pi}{8}\right]^{T}$. The reference position of the surface vessel was given as

$x_{d}(t)=\left[\begin{array}{l}10 \sin (0.1 t)(\mathrm{m}) \\ 10 \cos (0.1 t)(\mathrm{m}) \\ -0.1 t(\mathrm{rad})\end{array}\right]$.

Remark 3. The control gain matrices $C$ and $K$ are required to be chosen greater than the bounds of the uncertainties and must comply with the selection region given in (76). To address this issue, in Bidikli et al. (2013) and Bidikli et al. (2014), we designed a self-tuning algorithm for the similar type of the controllers in this paper. As a result, the controller gains in this paper are obtained via the selftuning algorithm in Bidikli et al. (2013) and Bidikli et al. (2014), thus avoiding the knowledge of bounds of the uncertainties. According to the proposed method in Bidikli et al. (2013) and Bidikli et al. (2014), controller gains are designed as sum of constant and time-varying parts and the positive selection of their constant parts is the only necessity of this method. When this necessity is ensured proposed method can appropriately deal with all bounds and the region definitions given in this paper.

Based on the self-tuning algorithm in Bidikli et al. (2013) and Bidikli et al. (2014), the controller gains were obtained as $K=\operatorname{diag}\{15,12.25,11.75\}$ and $C=\operatorname{diag}\{10,1.1,4.75\}$, and we chose $\alpha=3.44 I_{3}$.

The actual and reference positions and the position tracking errors are shown in Figs. 1 and 2, respectively. These results confirm that the proposed controller meets the tracking objective. The control inputs are shown in Fig. 3 to demonstrate the needed control effort during the control process. 

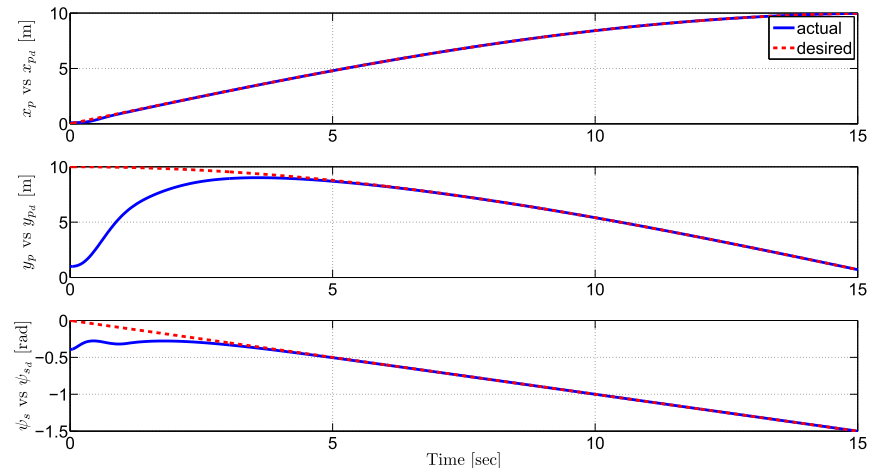

Fig. 1. Tracking results for noiseless case.
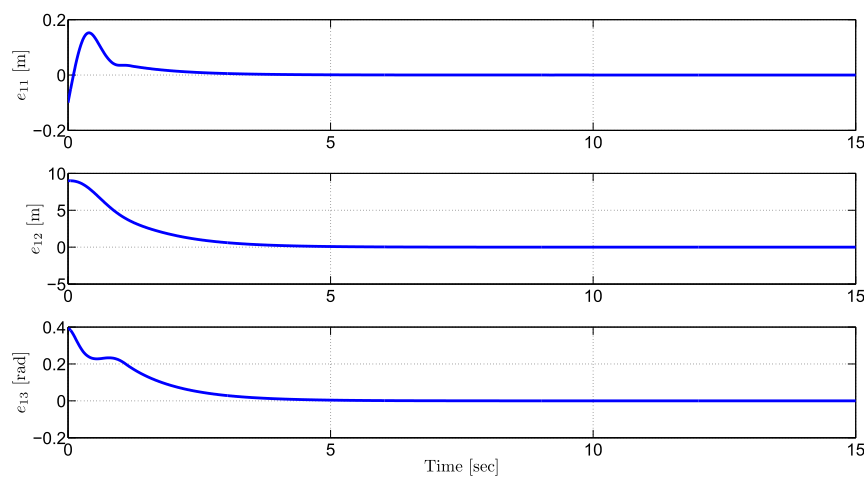

Fig. 2. Tracking error $e_{1}(t)$ for noiseless case.
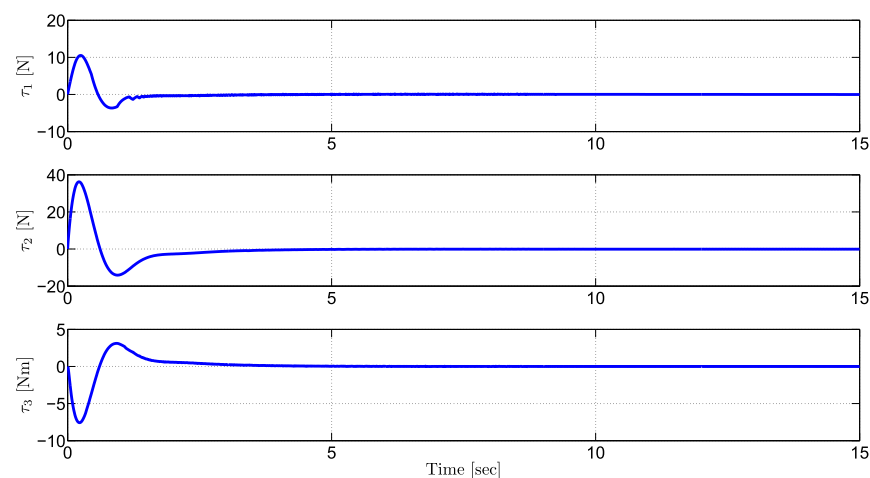

Fig. 3. Control input $\tau(t)$ for noiseless case.

Simulations were also performed by applying an additive zero mean Gaussian noise with $40 \mathrm{~dB}$ signal-to-noise ratio to the position and velocity measurements. Representing the possible environmental effects such as wind and waves and measurement noises and demonstrate the robustness of the designed controller against them are the main purposes of these simulations. Based on the self-tuning algorithm in Bidikli et al. (2013) and Bidikli et al. (2014), the controller gains were obtained as $K=\operatorname{diag}\{17.2,18.3,16.4\} \quad$ and $C=\operatorname{diag}\{13.5,12.6,12.5\}$, and we chose $\alpha=3.44 I_{3}$ for better comparison with the noiseless case.

The actual and reference positions and the position tracking errors are shown in Figs. 4 and 5, respectively. These results confirm that the proposed controller meets the tracking objective. The control inputs are shown in Fig. 6 to demonstrate the needed control effort during the control process.

Integral of the square of the norm of the tracking error $\int_{t_{0}}^{t}\left\|e_{1}(\sigma)\right\|^{2} d \sigma$ and the control input $\int_{t_{0}}^{t}\|\tau(\sigma)\|^{2} d \sigma$ were observed and recorded as performance measures for both cases. These results are
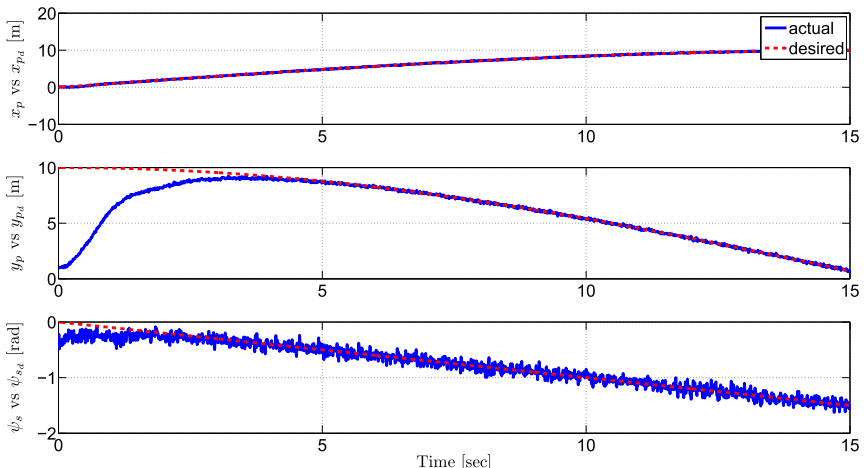

Fig. 4. Tracking results under the effect of noise.
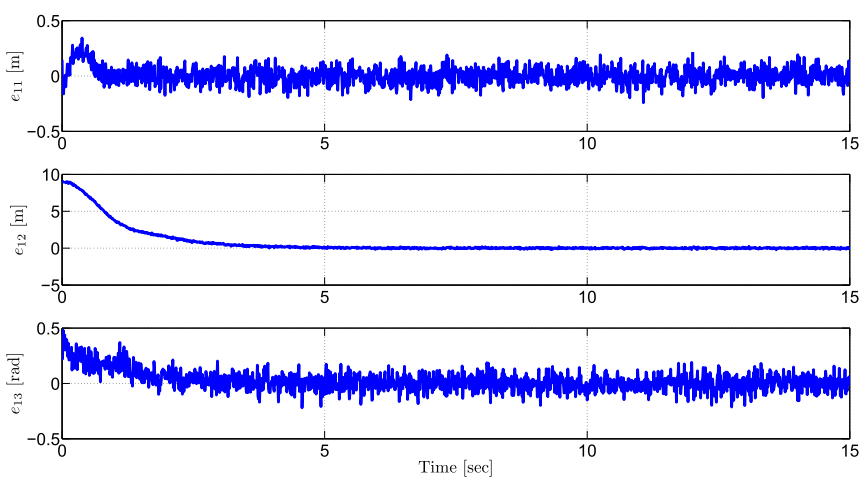

Fig. 5. Tracking error $e_{1}(t)$ under the effect of noise.
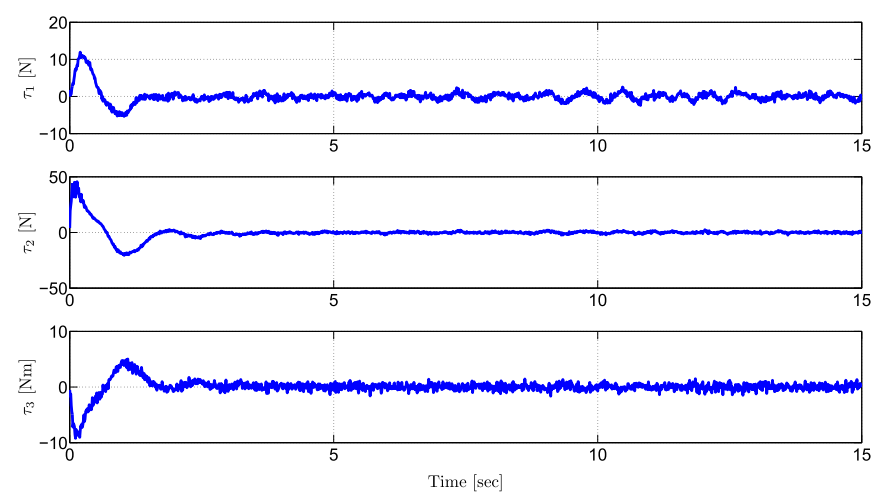

Fig. 6. Control input $\tau(t)$ under the effect of noise.

Table 1

Performance measures.

\begin{tabular}{lll}
\hline & $\int_{t_{0}}^{t}\left\|e_{1}(\sigma)\right\|^{2} d \sigma$ & $\int_{t_{0}}^{t}\|\tau(\sigma)\|^{2} d \sigma$ \\
Noiseless case & 62.64 & 544.33 \\
Under the effect of noise & 74.48 & 668.93 \\
\hline
\end{tabular}

presented in Table 1 to examine the simulation results numerically.

\section{Conclusion}

Robust control of dynamically positioned surface vehicles having non symmetric inertia matrices in their dynamics, is addressed. In addition to the uncertainties in the system dynamics, added mass terms are considered to be affecting the system at the acceleration level. A continuous nonlinear robust controller, that compensates the dynamical uncertainties and the asymmetry in the inertia matrix have been proposed when the input gain matrix has non-zero leading principle minors. We were able to obtain semi-global asymptotic tracking by using Lyapunov based arguments in 
conjunction with an integral inequality. The proposed methodology is backed up by extensive simulation studies.

\section{References}

Bidikli, B., Tatlicioglu, E., Bayrak, A., Zergeroglu, E., 2013. A new robust integral of sign of error feedback controller with adaptive compensation gain. In: Proceedings IEEE International Conference on Decision and Control, Florence, Italy, pp. 3782-3787.

Bidikli, B., Tatlicioglu, E., Zergeroglu E., 2014. A self tuning RISE controller formulation, In: Proceedings American Control Conference, Portland, OR, USA, pp. 5608-5613.

Bidikli, B., Tatlicioglu, E., Zergeroglu, Bayrak, A., 2013. An asymptotically stable continuous robust controller for a class of uncertain MIMO nonlinear systems, Tech. Rep. 1301.5483, ArXiv e-prints (Jan.).

Chen, J., Behal, A., Dawson, D.M., 2008. Robust feedback control for a class of uncertain MIMO nonlinear systems. IEEE Trans. Autom. Control 53 (2), 591-596.

Costa, R.R., Hsu, L., Imai, A.K., Kokotovic, P., 2003. Lyapunov-based adaptive control of MIMO systems. Automatica 39 (7), 1251-1257.

Do, K.D., Pan, J., 2005. Global tracking control of underactuated ships with nonzero offdiagonal terns in their system matrices. Automatica, 87-95.

Fossen, T.I., 1994. Guidance and Control of Ocean Vehicles. John Wiley and Sons, New York, NY, USA.

Fossen, T.I., 2011. Handbook of Marine Craft Hydrodynamics and Motion Control. John Wiley \& Sons, Hoboken, NJ, USA.

Fossen, T.I., Strand, J.P., 1999. Passive nonlinear design for ships using Lyapunov methods: full-scale experiments with a supply vessel. Automatica 35 (1), 3-16.

Fossen, T.I., 2002. Marine Control Systems: Guidance, Navigation, and Control of Ships,
Rigs and Underwater Vehicles, Marine Cybernetics AS, Trondheim, Norway.

Ihle, I.A.F., Jouffroy, J., Fossen, T.I., 2006. Formation control of marine surface craft: a Lagrangian approach. IEEE J. Ocean. Eng. 31 (4), 922-934.

Khalil, H.K., 2002. Nonlinear Systems 3rd edition. Prentice Hall, New York, NY, USA.

Kim, Y., Nam, B.W., Kim, D.W., Kim, Y.S., 2007. Study on coupling effects of ship motion and sloshing. Ocean Eng. 34 (16), 2176-2187.

Lee, D., Tatlicioglu, E., Burg, T.C., Dawson, D.M., 2008. Robust output tracking control of a surface vessel. In: Proceedings American Control Conference, Seattle, WA, USA, 2008, pp. 544-549.

Lee, D., Tatlicioglu, E., Burg, T.C., Dawson, D.M., Adaptive output tracking control of a surface vessel. In: Proceedings IEEE International Conference on Decision and Control, Cancun, Mexico, 2008, pp. 1352-1357.

Lewis, E.V., 1989. Principles of Naval Architecture Volume 3 Motions in Waves and Controllability, The Society of Naval Architects and Marine Engineers, Jersey City, NJ, USA.

Morse, A.S., 1993. A gain matrix decomposition and some of its applications. Syst. Control Lett. 21 (1), 1-10.

Newman, J.N., 1977. Marine Hydrodynamics. MIT Press, Cambridge, MA, USA.

Skjetne, R., Smogeli, O.N., Fossen, T.I., 2004. A nonlinear ship manoeuvering model: identification and adaptive control with experiments for a model ship. Model. Identif. Control 25 (1), 3-27.

Stepanyan, V., Kurdila, A., 2009. Asymptotic tracking of uncertain systems with continuous control using adaptive bounding. IEEE Trans. Neural Netw. 20 (8), $1320-1329$.

Techet, A.H., 2015. 2.016 Hydrodynamics (13.012), Massachusetts Institute of Technology Lecture. 〈http://ocw.mit.edu/courses/mechanical-engineering/2-016hydrodynamics-13-012-fall-2005〉. 